\title{
Design and Analysis of Elevator Control System Using PLC
}

\author{
Ganesh Nomula, Tharikoppula Sowmya, Gopi Gaddam
}

\begin{abstract}
A bring isone of the good sized angles in devices manage module in automobile software.For a remarkable many humans living in city groups, lifts have emerge as an crucial piece in their each day lifestyles. Basically said,an improve is a raising or bringing down thing, intended to deliver travelers or shipment, and is provided with a carand diploma that regularly moves in fixed aides and serves at the least landings.This paper typically

Centers round utilizing programmable reason controller to control the circuit and constructing the elevate version. HallEffect sensor is applied for the boost role. DC Motor is applied to control the during improvement of theelevator automobile. Push catches are carried out to name the boost vehicle. The raise function is portrayed through utilizing thedisplay unit. On this paper, automobile Station software program stepping stool motive software is implemented for 4 tales control machine.An carry framework is a vertical vehicle that efficaciously actions

Humans or products among floors of a shape. They're typically controlled by way of way of electricmotors. The most common carry is the rope boost. Inside the rope carry, the automobile israised and taken down via alternate with metal rope. Lifts additionally have electromagneticbrakes that connect to, while the automobile grinds to a halt. The electromagnetic truly keepsthe brakes in the vacant function. Rather than shutting them with the plan, the brakeswill truely brace close if the bring loses manipulate.
\end{abstract}

Catchphrases: percent, Elevator, DC Motor, TPLC Controller.

\section{INTRODUCTION}

Carry frameworks shape a category of DES's whose unpredictability makes them difficult to model,examine and enhance [10]. In a elegant bring framework, sorts of catches determine the increase development: lobby call catches and car name catches. The hall name catches are introduced at the board getting into the keep on the foyer of the shape, and the car call catches are delivered inside the raise. A increase framework has a couple of lobby name fastens on each ground, one for up corridor call and the alternative for down lobby call. While a hall name button is squeezed, the deliver moves to serve the foyer call proper away. In the wake of entering the raise, the traveler squeezes car call capture to choose his cause ground. The raise at that point climbs/all of the manner all the way down to the purpose floor. To increase use of the raise, the raise may additionally prevent at flooring that solicitation management of identical

Revised Manuscript Received on September 14, 2019.

Ganesh Nomula, Assis.Professor, Department of EEE, Siddhartha Institute of Technology \& Sciences, Narapally, Ghatkesar, Hyderabad, Telangana, India.

Tharikoppula Sowmya, Assist. Prof, Department of EEE, Siddhartha Institute of Technology \& Sciences, Narapally, Ghatkesar, Hyderabad, Telangana, India.

Gopi Gaddam, Assist. Prof, Department of EEE, Siddhartha Institute of Technology \& Sciences, Narapally, Ghatkesar, Hyderabad, Telangana, India. development bearings. The bring stops on the closest stated flooring for all covered solicitation. At the point while all calls are served, the improve stops and trusts that the subsequent call will show up, and the above interest rehashes.

Water driven and roped lifts are the 2 types of lifts getting used today. The principle plan contemplations forchoosing both electric footing power or water powered for a selected challenge are the quantity of flooring, the tallness of thebuilding, the quantity of people to be moved, desired traveller maintaining up instances and recurrence of utilization.

This venture is to shape and increase a boost utilising a programmable motive controller. Foyer impact sensor isused to understand the lift position. Pressure driven and roped lifts are the 2 varieties of lifts being used these days.Elevators are pervasive all through numerous staggered systems.

The number one reference convey become concocted via Archimedes in 312. Fromsome schooling supply, elevate were created as hyperlink on a hemp rope and poweredby hand or via thru creatures. This shape of raise modified into introduced within the SinaiMonastory of Egypt. Inside the seventeenth century, the rather little type lifts had been placed inthe strolling of england and France. In 1793, LvanKuliben made a lift withthe screw lifting issue for the wintry weather spot of Saint Petersburg. In 1816, anelevator modified into set up in the primary shape of Sub-moscow town calledArkhamgelskoye. Inside the middle 1800's, there have been many form of curd lifts thatcarried cargo. The general public of them ran the use of pressurized water. The foremost pressure pushed lifts utilized aplunger underneath the automobile to elevate or lower the elevate. A siphon implemented water strain toa plunger, or metallic segment, indoors a vertical chamber. In 1852, Elisha Otis introducedthe health increase, which predicted the autumn of the taxi, if the hyperlink broke. In 1857March twenty third, the primary Otis visitor raise became brought in new york metropolis. The firstelectric increase have become labored with the resource of Werner Von Siemens in 1880.

In 1874, J.W. Meaker covered a strategy which allowed elevatordoors to open and near securely. In 1882, whilst strain driven electricity was a well establishedtechnology, an organisation later named the London Hydraulic power business enterprise wasformed. In 1929, Clarence Conrad Crispen, with Inclinator agency of america,created the precept private elevate. 
Brief and clean p.C control The object of a percent check device is to 'counterfeit out' the contribution to a p.C so the developer can test and study the program earlier than set up order into it is walking condition. Our patent pending p.C take a look at systems accomplish this by means of the use of mounting at the contemporary terminal phase of the \% card and giving simple controls to turn advanced contributions on/off or exchange smooth signal. Inside the occasion that you are a expert who applications p.C. Or maybe a professional wanting a fast approach to test a p.C usefulness then these gadgets are for you. Spare time, cash and shame by using manner of solving troubles before they start. These p.C test systems are to be had to be bought in our gadgets section.

\section{ELEVATOR GADGET EVALUATE}

Raise vehicle moves it each upward ordownward heading. The advent sensor identified the advent of the elevate to therespective floor. Floor button is implemented to take the increase to the separate floor. Floorlamp suggests the signal of floor and route slight indicates the heading of elevatormovement, no matter whether it's miles upward or descending bearing. Carry button is implemented formoving the elevate vehicle each upward and descending manner.

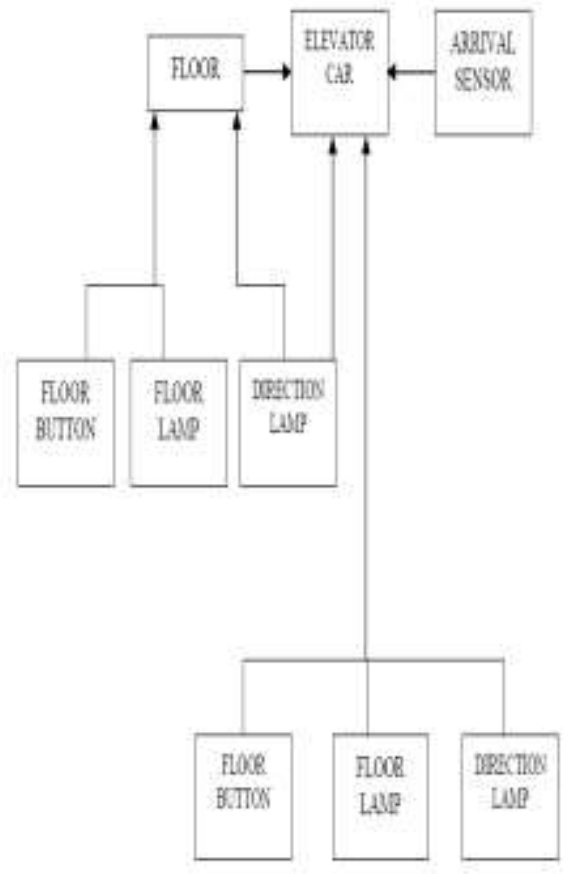

Fig1.1 Elevator System overview

Based theelevator transfer is squeezed, the raise automobile both above and downwarddirection. D.C. The engine is a good sized phase of the lift framework. Based onthe switch is squeezed, the DC engine both push in advance and invert tomove lift either upwards or downwards heading. Elevatorsystem entryway is one of the good sized elements of the lift framework. At the point whilst the lift automobile halted inparticular ground, the carry entryways opened to vacationers who will turn out andcome into the carry. The appearance of the sensor applied in each ground, to apprehend theelevator automobile. At the factor while a specific automobile to a selected floor is come to, this look sensordetects lift automobile and the vehicle halted.
At the point while the purchaser presses the raise button, elevate catches sendsthe sensor carry demand capture to the framework, recognizing the goal ground userwishes visits. At the point while another solicitation comes, the new solicitation is introduced to the rundown offloors visits. In the event that the raise is stationary, the framework figures out in which directionthe framework ought to pass in an effort to serve consequent solicitations. Commandsthe framework elevate entryways close, whilst the purchaser presses the catch the carry entryway shut. Whenthe entryway is shut, the framework directions the engine to show over moving the lift, each above and under the bearing, in view of a switch pressed. When elevate moves among floors, the lift thatthe sensor distinguishes the arrival of shifting in the direction of the floor and recommend the framework to forestall the carry entryway of the carry framework andopen.

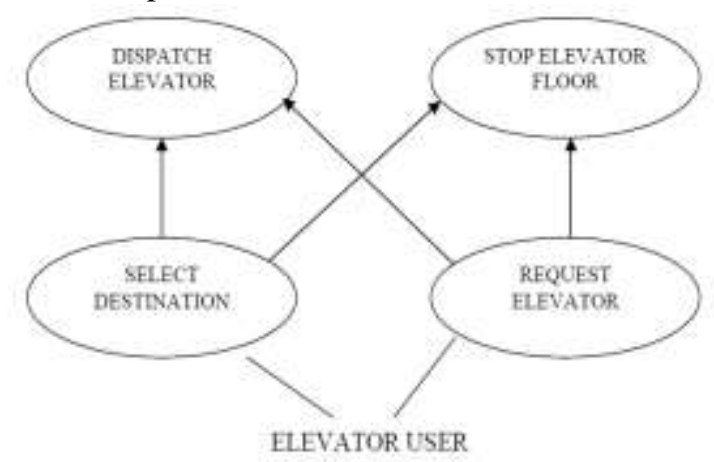

Fig.1.2 Elevator Dispatching Strategy

\section{PROPOSED SYSTEM \& RESULTS}

A surmised little scale deliver version with percent is acquired for thecontroller shape which utilizes the Ladder Language depending at the GE FANUC VersamaxPLC.The stepping stool purpose hasimplemented with the useful resource of making use of VersaPro. 2.02.In this manipulate shape method, the mild structured Resistor (LDR) is usedto experience the lift ground.GE FANUC Versamax \% is having a configurable memory of $64 \mathrm{kbyte}$. The authorprovided to enhance the superb in raise frameworks, create and drives the applied setting and increment the reliabilityof raise. The creator moreover referenced to accomplish fast nine-diploma everlasting Magnet Synchronous MotorControl device.The a ways off module to understand the transmission of purchaser records,percent control framework are acquired, the sequential correspondence mode between the $\%$ and the faraway module wasadopted.SI4432 Transceiver, single-Chip computer and percent are applied to adjust the control association of constructionelevator.The manipulate association of the development convey have become constructed from \%, faraway calling machine and wirelesshost handset. Lift manipulate framework can determine the following travel heading depending on the call statistics andthe current-day hobby without a doubt, recognizes unattended interest.

Published By: Blue Eyes Intelligence Engineering 
Using satiate outline to the displaying, plan and execution of anelevator framework, whose framework conduct consists of amassing multifaceted nature of kingdom portrayals, and inconvenience ofunderlying control approach. In view of the operational improvement of a lift, they infer the associated kingdom graph modelby investigating the inborn various leveled shape of the elevate. This exploration become upheld to a few degree through the grantNSC90-2213-E-011-020 and NSC90-2212-E-014-023 by way of the countrywide science Council, Taiwan, R.O.C.

\section{Statecharts}

The statecharts technique was furnished as a visualformalism for indicating the conduct of complex reactivesystems [7]. As inside the traditional $u$. S .transitiondiagrams, the belief of states contains the crucial componentof statecharts. Anyways, states is probably embeddedinto superstates in the long run making pecking orders of states. Superstatesmay be taken into consideration one in all kinds: AND-states orXOR-states. The preceding catches the concept of independence,e.G. Picture and sound might be simultaneously in awebsite. The very last compares to refinement of states,e.G. A mild each remains on OFF or ON united states of america. Thecomponents of an AND-country are known as symmetrical componentsand have the unmistakable element that a framework inan ANDcountry is likewise inside the entirety of its symmetrical components.Because of this a kingdom $\mathrm{S}$ is depicted as comprising of twoor more and more symmetrical segments, and to be in kingdom $\mathrm{S}$ entailsbeing in the ones segments all the on the equal time as. Thenotation is spoken to via a ran line that segments thestate into its factors. On the other hand, if the framework is in aXOR-country, it must be in only one in every of its substates.States join via adjustments, which comprise ofthree critical elements: an event articulation, a condition, andaction proclamations.

\section{ELEVATOR manage the use of \%}

The essential Programmable good judgment Controller, $\%$ grow to be created with the useful resource of a gathering of professionals at popular vehicles in1968. It grow to be created even as that enterprise employer have become attempting to find a desire to supplant complicated hand-off manipulate system.The time period 'programmable reason controller' is characterised with the aid of EN 61131-1 as a carefully strolling virtual framework whichuses a programmable memory for the inner stockpiling of patron located guidelines for actualizing specificfunctions, for instance, motive, sequencing, timing, checking and range juggling to manipulate thru automated or smooth inputsand yields, exceptional machines or technique.

Stepping stool graph is a programmed manage chart language that created all through world war II. Stepping stool reason is theprimary programming language of programmable cause controllers. For the reason that \% became created to supplant relaylogic manage frameworks, it modified into sincerely feature that the underlying language carefully looks like thediagrams used to record therelay cause. By using this system, the experts and experts using the early p.C. Did no longer require retraining tounderstand the program. To present stepping stool purpose programming honest switch circuits are changed over to switch logicand then to percent stepping stool cause. Any manage undertaking modifications are finished thru converting this gadget. This is the reason the useof the \% is desired to the customary difficult confused out circuits in modern-day controls.

There are numerous kinds of programming dialects in Programmable good judgment Controller, \%. Dialects aretypically consistent to Ladder common sense (LD), Sequential characteristic Block (SFC), function Block Diagram (FBD) andStructure textual content (ST). The normal software language of \% is stepping stool chart.

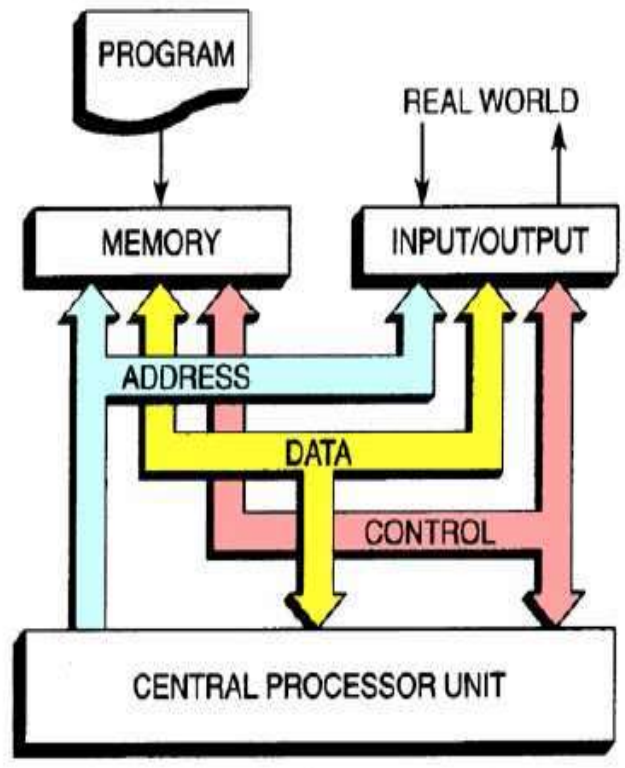

Fig.1.3 PLC Architecture

So as to design a control circuit, it is partitioned into various gadgets or modules for its exact task or controlwhich first might be tried or actualized autonomously after which combined. The premise of PLC basedelevator oversee might be classified into three head associations. The first is the floor, the second is the PLC controller andthe last is the Elevator. The square chart of PLC Based Elevator Control System is portrayed in Figure 1.Three Toaccomplish the PLC based absolutely control device, the design utilizes six prevalent added substances: PLC controller, DC engine rationale force,push button, degree sensor, show unit and Elevator. Level sensors are utilized to perceive the lift job and pushbuttons are was input by means of the customer demand. The presentation unit will show the scope of ground. The PLCcompares the individual solicitation and the surge catch to control the lift engine Up or Down. At the point when the purchaser demand isgreater than the sensor cost, the engine will cross up and it's miles not exactly the sensor expense, it will cross down. At the point when the twovalues are equivalent, the engine must anticipate. 


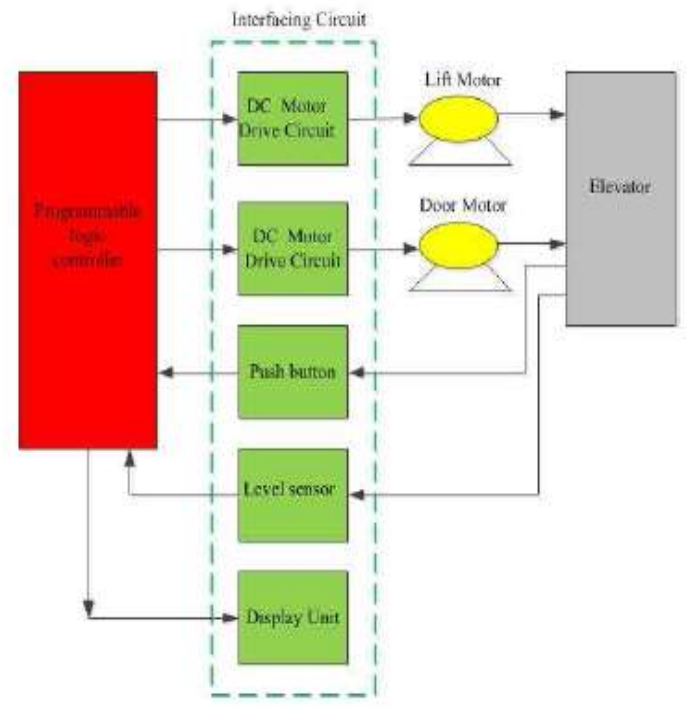

Fig.1.4 PLC Based Elevator Control System

This format can be separated into a few gadgets or modules. They are the sensor handling unit and power unit unit.There more than one gadgets and added substances utilized inside the design to authorize each unit. The gadget is used in thissystem are as per the following;

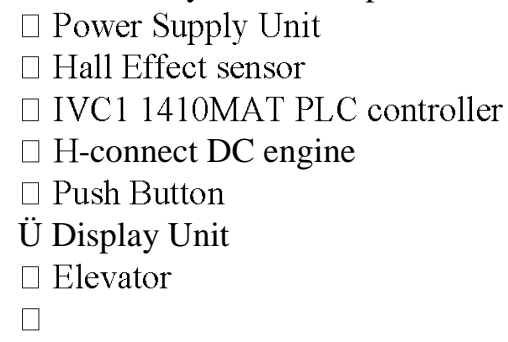

\section{A. Power Supply Unit}

DC power supply unit is vitalcomponent in present day advanced gadgets since they need a determination of DCvoltages for their tasks. The explanation of the vitality supply is to offer the significant measure of vitality specifiedvoltage from essential resources.

\section{B. Depiction of Hall Effect Sensor}

A Hall Effect sensor is a transducer that shifts the yield voltage in response to the attractive zone. Effectsensors passageway is utilized for separation, position, speed and contemporary detecting location packages.In its best structure, thesensor works as a simple transducer, quickly lower back pressure. A Hall Effect sensor might be utilized during the current second measurethe without intruding on the circuit. Regularly, joined with a circuit that enables the gadget to act adigital mode (on/off) and can be called switches. It is commonly utilized for a haggle speeds, suchas for an inward burning motor start timing, tachometers and antilock braking contraption. It basically is made ofa thin piece out of p-kind semiconductor material square shape. A conventional Hall Effect sensor has 3 wires or terminals; one for floor, one for the stock or reference voltage and one for yield. To create the yield sign, need to besupplied with a voltage of five to twelve $\mathrm{V}$. There are explicit sorts of attractive movement, together with Head-on, Sideways, push-force and push-motivation discovery.

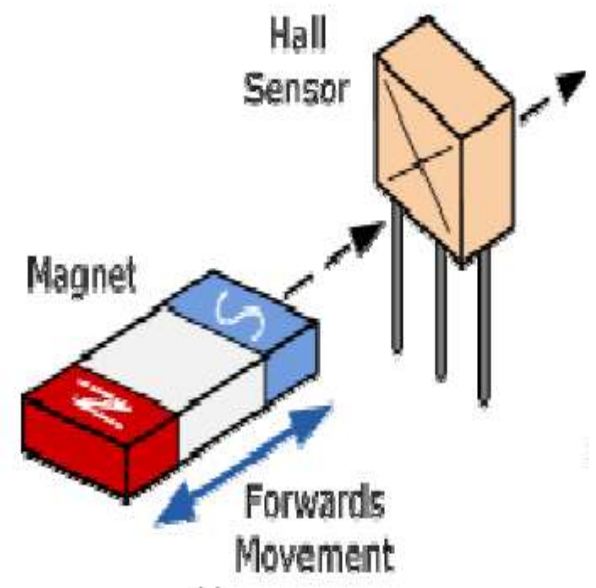

Fig.1.5 Heard-on detection

\section{Features of IVC1 1410MA TPLC Controller}

The programmable controller PLC used in this look at is the IVC As proven in Figure 6, Port0 and PORT1 is for conversation. Port0 is RS232, and use the Mini DIN8 socket, while PORT1 is the RS485 or RS232. Bus socket is to attach the extension module. Switchcan mode selector is ready to $\mathrm{ON}$ or $\mathrm{OFF}$.

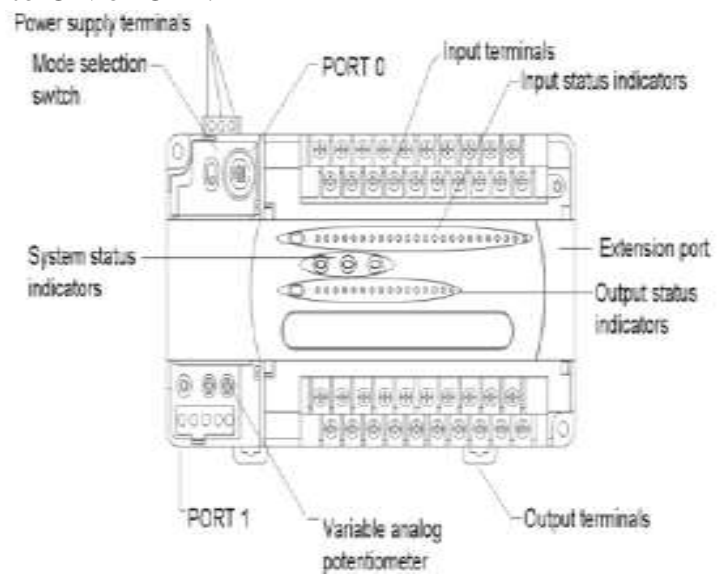

Fig.1.6Structure of IVC1 series basic module

\section{H-bridge DC Motor}

Bidirectional control of a DC engine requires a circuit called a Happearance, is equipped for pass forefront both way by means of the engine winding.This contraption. It is known as a "Hlike a $\mathrm{H}$ letter. A H-connect is an electronic circuit which licenses electric cars to be run ahead or in reverse course. It is as a rule utilized in engine control of lift. It is to be had as incorporated circuits or might be developed fromseparate segments for specific format. In this gadget, the DC engine with Hadvance the lift vehicle to the consequent job. The engine can offer for every guidance: clockwise (CW) directionand counterclockwise (CCW) way. This circuit utilizes 4 transistors for ahead and inverse directions. Itsoperation is as per the following. To pivot the DC engine (a) Heard-on location (b) How Hall Effect worksIVC1-1410MAT. It has 14 information sources and 10 outputs.The $\mathrm{H}$-connect, named for its schematicH-connect topology transformed into chosen inH-connect rationale

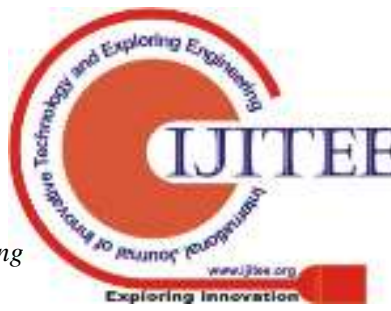


power circuit is utilized to four transistors are utilized. At the point when the transistor $\mathrm{Q} 2$ and $\mathrm{Q} 3$ are $\mathrm{ON}$ and different transistors are OFF, the voltage all through themotor will be horrendous, permitting counterclockwise activity of the engine. In DC engine, clockwise course for Upconditionand counter clockwise bearing is for Downcircumstance.

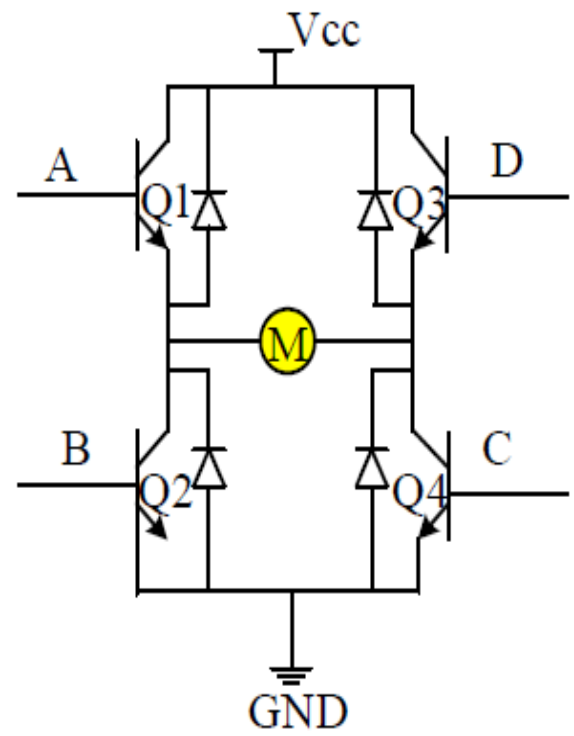

Fig.1.7 H-bridge DC-motor Circuit

\section{E. Results}

Recreation of a lift position manage is completed with the Auto Station to determine theperformance ofthe controller.AutoStationsoftware stage used to steer checks. PLC's preliminary comes

withIVC1-1410MAT

programmable rationale controller.In this framework, theladder software ought to be run in programming download controllerby PLC stepping stool.

The critical conditions are moreover required;

\section{$\square$ PLC \\ $\square$ Programming Device Cable Connectors \\ $\square$ Programming Software}

\section{END}

Hand-off generally applied and the IC board has been supplanted through a PLC to control ofmachines easy and reasonably-priced to use on this carry. By building up this proposed framework, the aftereffect of the lift manipulate framework can beapplied in reality. By utilising the raise manage framework dependent on PLC, the ideal position can be evaluated. That

The reenactment outcomes of a four-tale framework has discussed.As an occupation later on, the model basedelevator IVC1 1410MAT PLC is planned to bring together and check to be implemented in future paintings global.In actual dependent on the operational move ofan lift, we collect the associated Model statechart bylooking to the feature diverse leveled shape of elevator.The favored role of the proposed method is clearpresentation framework conduct and status for utilization.

\section{REFERENCE}

1. Yaing Sun, "Encouraging Module Design of Elevator Controlled through PLC" smaller scale APPLICATION, 2013, pp.63-67, 31 (five).

2. Jie Zhang, "The use of PLC within the elevate manipulate framework", Journeel of Liaoning Normal University (Natural Science Edition), 2009, pp.318320,32 (three).

3. Darshil, Sagar, Rajiv, Pangaokar and S.A. Sharma "Based Elevator System Development PLC with Color Sensing Capabilities for Industrial Material Handling Plant", Jiont International Conference on Power System Technology and IEEE Power India Conference, 2008, pp.1-7

4. Jayawardana.H.P.A.P., Amarasekara. H.W .K.M., Peelikumura.P.T.S., Jayathilaka.W.A.K.C., Abeyaratne.S.G. AndDewasurendra.S.D. "Plan and implenentation of astatechart based totally reconfigureurable lift controller", 6th IEEE International Industrial and Information Systems, IEEE Conference Publications, pp. 352-357

5. Pillay, P., and Krishnan, Modeling DC Motor Drives. IEEE Trans. Modern Applications, 1988, p. 537-541, Vol. 35.No.4. IEEE Database.

6. Zhang Yajun, Chen Long, Fan Lingyan, "A Design Elevator Positioning Control System Model," IEEE Int. Systems and Signal Processing Conference, Zhenjiang, China, June 8 to 10.2008 , p. 535-538.

7. Hongqun Li, Yue Zhou, PLC Control and Real-time gazing of Sightseeing Lift.Techniques Automation and Applications 2008, Vol.27 \# 11.

8. Liu Xiaojuan, Elevator Development System Based on Fieldbus Monitor, February 2008, Vol.30 No.1. Diary of EEE.

9. S.B. Ron Carter, "Structure and Implementation of PLC primarily based Elevator", April 2013, Volume 68_ 7. 